\title{
System Engineering of Emergent Serum Therapy to Combat COVID-19 and Other Pathogenic Pandemics
}

\author{
Peter K. Law \\ Cell Therapy Institute, Wuhan, China \\ Email: peter@celltherapy.com
}

How to cite this paper: Law, P.K. (2020) System Engineering of Emergent Serum Therapy to Combat COVID-19 and Other Pathogenic Pandemics. Open Journal of Regenerative Medicine, 9, 8-14. https://doi.org/10.4236/ojrm.2020.91002

Received: March 19, 2020

Accepted: March 20, 2020

Published: March 23, 2020

Copyright $\odot 2020$ by author(s) and Scientific Research Publishing Inc. This work is licensed under the Creative Commons Attribution International License (CC BY 4.0).

http://creativecommons.org/licenses/by/4.0/

\begin{abstract}
Life-saving procedures of an emergency protocol to combat fatal ambushes of viral or bacterial pathogens are listed below for immediate dissemination and implementation. This unique protocol of serum therapy commands the highest benefits-versus-risks ratio, and is safe, efficacious, user-friendly and inexpensive to implement. A formal evaluation is presented to support upgrading convalescent plasma therapy to serum therapy in the context of presenting fewer donor antigens, thus evoking less lethal sensitization to obtain greater safety and efficacy in treatment.
\end{abstract}

\section{Keywords}

Serum Therapy, Plasma Therapy, COVID-19, Coronavirus, Vaccine, Antibody Medicine, Transfusion Immunology

\section{Introduction}

According to national headline news reported every morning in China, as of March 18, 2020, 198,127 people have been diagnosed with COVID-19 around the world with 7976 deaths and 107,376 sick survivors. Of the 82,775 rehabilitated patients, $84.3 \%$ are in China. Non-Chinese deaths have already exceeded Chinese deaths by 1492, and the death toll is rapidly rising, specifically in Italy and Iran.

Beginning at around February 14, non-Chinese cases are increasing exponentially and have no sign of plateauing for at least four months according to previous Chinese experience. It is long-overdue for WHO and national health organizations to implement scientifically superior protocols of therapies that are available to save human lives. 
According to press conference reports of the National Health Commission of the People's Republic of China (NHCPRC), COVIDS-19 in China is characterized by having $85 \%$ of its patients, usually between the ages of 15 to 55 , exhibiting mild symptoms that disappeared within two weeks of treatment with antibiotics, steroids or the Chinese herb of Ban Lan Geng. These patients were in good general health before being contaminated, and had spontaneously developed antibodies against the Coronaviruses and their antigens. These antibodies were found in the lymph, serum, plasma, and whole blood in good titers of the rehabilitated individuals.

Without considering the complete implication of transfusion immunology, especially when no approved viral medication readily available, researchers often grasped convalescent plasma as the last straw because of its simplicity in usage and needing no FDA/EMA/CFDA regulatory approval. On February 19, 2020, the NHCPRC, on the recommendation of academicians and experts, announced that convalescent plasma was adopted as one of the treatment programs for COVID-19.

However, in the 2014 systematic review and exploratory meta-analysis by a panel made up of essentially WHO experts, it was concluded that, "Convalescent plasma may reduce mortality and appears safe. This therapy should be studied within the context of a well-designed clinical trial or other formal evaluation...". The authors stated in the Results, "We identified 32 studies of SARS coronavirus infection and severe influenza. Narrative analyses revealed consistent evidence for a reduction in mortality, especially when convalescent plasma is administered early after symptom onset". "Studies were commonly of low or very low quality, lacked control groups, and at moderate or high risk of bias" [1].

It appears that formal evaluation is deemed necessary, prioritizing serum therapy over plasma therapy in the context of presenting less foreign antigens, thus evoking less allogeneic sensitization and providing greater safety and efficacy in treatment. This is in the same context of prioritizing plasma therapy over whole blood transfusion in viral encounters because of better transplantation immunology.

\section{Formal Evaluation}

Both serum therapy and plasma therapy are passive immunotherapies that allogeneic sensitization must be reduced to a bare necessity to avoid allergic or fatal adverse reactions. This is the guiding principle of transplantation immunology. Any deviation can be fatal, leaving the critically- or terminally-ill patients dead by acute respiratory distress syndrome (ARDS) and multiple organ failure. A formal evaluation of the two therapies is listed below.

1) Both convalescent serum and plasma contain the antiviral antibodies, and as such, both can be useful to combat COVID-19. However, by definition, plasma is composed of albumin, fibrinogen and numerous clotting factors. These allogeneic antigens produce blood clots when exposed to the recipient's platelets 
during plasma transfusion. These are sensitizing epitopes that must be removed to avoid future complications and ARDS. I shall not elaborate further on the seriousness of overloading the host immune system with a large amount of fibrinogen as previously reported. Its significance cannot be underestimated because it usually leads to death of the critically- and terminally-ill patients [2]. The presence of various allogeneic clotting factors in the host blood stream will lead to graft-versus-host reactions that cause allergy and overt immunologic rejection. Why insist on having these potential risks when it is so easy to switch to serum therapy? Serum, by nomenclature, is the remain of plasma after the clotting proteins and factors are removed.

In the pioneering development of gene therapies and cell therapies treating Duchenne muscular dystrophy [3]-[7], ischemic cardiomyopathy [8]-[13], Type II diabetes [14] [15] [16] [17], cancer [18] [19] and aging disfigurement [13] [17] [19] spanning nearly 30 years, host serum has always been the medium of choice to carry allogeneic human myoblasts into the recipients. The simple but effective procedures of serum extraction were designed to be used in areas where social economy prevails far less behind the devastation of COVID-19.

\section{Serum for Therapy}

After meeting the Inclusion/Exclusion criteria that are specific for different country, religion and denomination, and signing of the Donor Informed Consent, whole blood was drawn slowly and steadily under GLP conditions by a certified phlebotomist from the median cubital vein of the rehabilitated volunteer donor through a 21 -gauge butterfly needle taped in place. Four $60-\mathrm{mL}$ syringes each containing $3 \mathrm{~mL}$ of anticoagulant citrate dextrose solution are used to collect approximately a total $240 \mathrm{~mL}$ of whole blood. Depending on the physical condition of the donor, the procedure can be repeated in two weeks.

Under GMP conditions, the blood collected is distributed evenly into four 60 $\mathrm{mL}$ conical glass centrifuge tubes of a refrigerated centrifuge maintained at $4^{\circ} \mathrm{C}$ to $8^{\circ} \mathrm{C}$. Centrifugation was maintained at $2000 \mathrm{rpm}$ for 15 minutes [19]. The supernatant is pipetted out and transferred immediately with a sterile pipet-aid into a sterile $300 \mathrm{~mL}$ flask under a Biologic Safety Cabinet with no air exchange in both directions. The precipitate of blood cells in each tube was mixed with 50 $\mathrm{mL}$ of ice-chilled saline, vortexed for 10 minutes, aspirated and injected slowly with a syringe into the donor's blood stream through the same route of phlebotomy, the median cubital vein. Care must be taken not to introduce any air in the system.

The flask of nearly cell-free plasma is put under a strong ultra-violet light for 4 hours and then stored overnight in a refrigerator maintained at $4^{\circ} \mathrm{C}$ to $8^{\circ} \mathrm{C}$. On the next day, the clear serum overlying the soft dark red clot is careful pipetted out and force-filtered through a silicon 0.22-micrometer filter with a power pump. The clear filtrate with a mild yellow color is the antiserum for transfusion. A 3-mL test sample is pipetted out to confirm absence of HIV, HAV, HBV, $\mathrm{HCV}, \mathrm{CMV}$, syphilis, bacteria, mycoplasma, and endotoxin for quality assur- 
ance. Another $0.5 \mathrm{~mL}$ sample is also taken for record keeping. It will be chilled to $-80^{\circ} \mathrm{C}$ overnight, then transferred to the cryo-tank storage with liquid nitrogen.

ELISA tests will be conducted to determine absence of viral antigens and potency of the antibodies. According to CNBG, $95.4 \%$ of the donors showed titers of antiviral antibodies IgG, ranging from $68 \%$ of the plasma collected having high titers $>1: 320 / \mathrm{mL}$ and moderate titers $>1: 160 / \mathrm{mL}$, to $31.7 \%$ at low or no titers $<1: 160 / \mathrm{mL}[20]$.

All samples of antisera with the same blood type will be pooled to include greater numbers and quantity of antibodies.

\section{Serum Therapy}

Serum therapy will produce the best results when administered between the first and the second week of infection. This is to provide passive immunogens leading the host's neutralizing antibodies/IgG production against lesser quality and quantity of viral antigens. Towards this end, early diagnosis and treatment are crucial before the disease advances to the critical stage. This does not mean that serum therapy is not effective in critically- and terminally-ill cases. In fact, it is specially designed for them.

After meeting the Inclusion/Exclusion criteria that are specific for different countries, religion and denomination, and signing the Treatment Informed Consent, patients receive serum therapy from Board-certified physicians under GCP conditions. All participants are properly gowned, masked and hooded, with gloves and shoe-covers, to prevent infection. A certified phlebotomist gains access to the recipient's median cubital vein, while transfusion of the processed serum is conducted by at least one Board-certified physician, accompanied by a cardiopulmonary specialist and two nurses. $\mathrm{ABO}$-compatible identical antiserum having moderate titers or above will be injected slowly at $5 \mathrm{~mL} / \mathrm{kg}$ body weight of the patient. The treatment procedure can be repeated on the next day if needed.

2) Post-transfusion mortality among recipients of $A B O$-compatible but nonidentical plasma is a common, inadvertent event associated with convalescent plasma transfusion. In the world's largest study of 86,082 Swedish patient records obtained from the SCANDAT database with the patients receiving their first plasma transfusion between 1990 and 2002, 24\% was exposed to compatible non-identical plasma. In emergency situation such as COVID-19, exposure was inadvertent, and exposure rate had to be higher. The Swedish group of exposed patients demonstrated, in a 14-day-follow-up study, an increased mortality of about $10 \%$ as a result of non-identical blood type matching, with higher risks in group $\mathrm{O}$ recipients, especially when the compatible plasma was from group $\mathrm{AB}$ donors. This increase in mortality of 1033 deaths is highly significant considering the large population of patients involved. Circulating immune complexes (CICs) were implicated as the cause of death [21]. Patients who received ABO-compatible nonidentical plasma also had a significantly higher incidence of ARDS [22]. In emergency lifesaving resuscitation, the risk of hemolytic trans- 
fusion reactions from transfusion of group $\mathrm{O}$ blood to nongroup $\mathrm{O}$ recipients constitutes risk that is outweighed by the benefits [23]. I had previously emphasized the need for direct blood type matching in the proposed serum protocol [2].

3) There were concerns expressed over lack of donors to provide sufficient plasma. With 69,739 patients already rehabilitated, and 8182 still sick in China, there is plenty of plasma available. Sizeable stocks of $A, B, O, A B+$ and $A B$-antisera can be obtained by pooling independent samples of the same blood type to include all antiviral antibodies to maximize the potency of the antisera for neutralizing the viral antigens from different mutations.

4) COVID-19 in China is likely to subside to a minimum by the end of March as predicted [2]. Death toll on March 17, 2020 was 11. After treating the 2622 critically-ill and terminally-ill patients most of whom are over the age of 60 , China should have enough antisera to treat non-Chinese cases until there is enough donated plasma from rehabilitated patients overseas.

5) Manufacture technology of IgG and IgG fragments are now available if $50 \mathrm{~L}$ of antisera pooled from all blood types can be spared for this specific purpose. Accordingly, if each of the rehabilitated individual that has not donated would donate $100 \mathrm{~mL}$ of their precious blood towards that end, there will be opportunity to produce highly purified whole IgG and IgG fragments according to the WHO guidelines for the production, control and regulation of IgG published in year 2010. Whole IgG against SARS coronavirus was found present in the patient's blood 3 years after rehabilitation [24].

This will take time. For now, it is serum therapy.

\section{Conclusion}

This is the first report to have documented plausible mechanisms and transfusion immunology in support of the emergent use of serum therapy to combat COVID-19 and future viral or bacterial pathogenic pandemics. The safety and efficacy that it demonstrated, though preliminary, lead the way to develop a new treatment for infectious diseases. Lacking graft-versus-host reactions, convalescent antisera and their IgG derivatives are safe biologics. Apart from this scientific treatise, well-designed randomized, double-blinded clinical trials have to be conducted at their earliest comparing the safety and efficacy of serum therapy versus plasma therapy.

\section{Acknowledgements}

P. K. L. is a National Distinguished Expert of CHINA. This work was supported by the Cell Therapy Institute, Wuhan.

\section{Conflicts of Interest}

The author declares no conflicts of interest regarding the publication of this paper. 


\section{References}

[1] Mair-Jenkins, J., Saavedra-Campos, M., Baillie, J.K., et al. (2015) The Effectiveness of Convalescent Plasma and Hyperimmune Immunoglobulin for the Treatment of Severe Acute Respiratory Infections of Viral Etiology: A Systematic Review and Exploratory Meta-Analysis. The Journal of Infectious Diseases, 211, 80-90. https://doi.org/10.1093/infdis/jiu396

[2] Law, P.K. (2020) Emergent Serum Therapy and Antibody Medicine to Counteract Sudden Attacks of COVID-19 and Other Pathogenic Epidemics. Open Journal of Regenerative Medicine, 9, 1-7. https://www.scirp.org/journal/ojrm https://doi.org/10.4236/ojrm.2020.91001

[3] Law, P.K., Bertorini, T., Goodwin, T.G., Chen, M., Fang, Q.W., Li, H.J., et al. (1990) Dystrophin Production Induced by Myoblast Transfer Therapy in Duchenne Muscular Dystrophy. The Lancet, 336, 114-115. https://doi.org/10.1016/0140-6736(90)91628-N

[4] Law, P.K. (1992) Myoblast Transplantation. Science, 257, 1329-1330. https://doi.org/10.1126/science.1529326

[5] Law, P.K. (1993) Myoblast Transfer Therapy. The Lancet, 341, 247. https://doi.org/10.1016/0140-6736(93)90115-W

[6] Law, P.K., Goodwin, T.G., Fang, Q., Duggirala, V., Larkin, C., Florendo, J.A., et al. (1992) Feasibility, Safety, and Efficacy of Myoblast Transfer Therapy on Duchenne Muscular Dystrophy Boys. Cell Transplantation, 1, 235-244.

https://doi.org/10.1177/0963689792001002-305

[7] Law, P.K., Goodwin, T.G., Fang, Q., Vastagh, G., Jordan, T., Jackson, T., Kenny, S., Duggirala, V., Larkin, C., Chase, N., Phillips, W., Williams, G., Neel, M., Krahn, T. and Holcomb, R. (1998) Myoblast Transfer as a Platform Technology of Gene Therapy. Gene Therapy and Molecular Biology, 1, 345-363.

[8] Law, P., et al. (2000) World's First Human Myoblast Transfer into the Heart. Frontiers in Physiology, A85.

[9] Law, P.K., et al. (2003) Myoblast Genome Therapy and the Regenerative Heart. In: Kipshidze, N. and Serruys, P., Eds., Handbook of Cardiovascular Cell Transplantation, Martin Dunitz, Ltd., London, 241-257. https://doi.org/10.3109/9780203640685-18

[10] Law, P. (2006) Heart Cell Therapy Symposium. Journal of Geriatric Cardiology, 3, 131-180.

[11] Ye, L., Haider, H.Kh. Tan, R.S., Toh, W.C., Law, P.K., Tan, W.B., Su, L.P., Zhang, W., Ge, R.W., Zhang, Y., Lim, Y.T. and Sim, E.K.W. (2007) Transplantation of Nanoparticle Transfected Skeletal Myoblasts Overexpressing Vascular Endothelial Growth Factor-165 for Cardiac Repair. Circulation, 116, I113-I120. https://doi.org/10.1161/CIRCULATIONAHA.106.680124

[12] Ye, L., Haider, H.Kh., Tan, R.S., Su, L.P., Law, P.K., Wei, Z. and Sim, E.K.W. (2008) Angiomyogenesis Using Liposome Based Vascular Endothelial Growth Factor-165 Transfection with Skeletal Myoblast for Cardiac Repair. Biomaterials, 29, 2125-2137. https://doi.org/10.1016/j.biomaterials.2008.01.014

[13] Law, P.K. and Law, D.M. (2011) Human Myoblast Genome Therapies and Devices in Regenerative Medicine. Recent Patents on Regenerative Medicine, 1, 88-117. https://doi.org/10.2174/2210297311101010088

[14] Law, P.K., Law, D.L.M., Lu, P., et al. (2004) The World's First Myoblast Study of Type II Diabetic Patients. Business Briefing. North American Pharmacotherapy, 
No. 2, 361

[15] Ma, J.H., Su, L.P., Zhu, J., et al. (2013) Skeletal Myoblast Transplantation on Gene Expression Profiles of Insulin Signaling Pathway and Mitochondrial Biogenesis and Function in Skeletal Muscle. Diabetes Research and Clinical Practice, 102, 43-52. https://doi.org/10.1016/j.diabres.2013.08.006

[16] Ye, L., Lee, K.O., Su, L.P., et al. (2009) Skeletal Myoblast Transplantation for Attenuation of Hyperglycaemia, Hyperinsulinaemia and Glucose in Tolerance in a Mouse Model of Type 2 Diabetes Mellitus. Diabetologia, 52, 1925-1934. https://doi.org/10.1007/s00125-009-1421-9

[17] Law, P.K. (2016) Disease Prevention and Alleviation by Myoblast Transplantation. Open Journal of Regenerative Medicine, 5, 25-43. https://doi.org/10.4236/ojrm.2016.52003

[18] Law, P.K., Song, S.J., Lu, P., Gao, Y., Ao, M., Zhao, H., Bai, L., Guo, K. and Law, D.M. (2017) World's First Myoblast Treatment of Human Cancer Found Safe and Efficacious. Open Journal of Regenerative Medicine, 6, 1-16. https://doi.org/10.4236/ojrm.2017.61001

[19] Law, P.K., et al (2019) Myoblasts Provide Safe and Effective Treatments for Hereditary Muscular Dystrophies, Cardiomyopathies, Type 2 Diabetes, Solid Tumors and Aging. In: Haider, K.H. and Aziz, S., Eds., Stem Cells-From Hype to Real Hope, Walter de Gruyter GmbH, Berlin/Boston, 71-97. https://doi.org/10.1515/9783110587043-004

[20] Yang, X.M. and Hou, J.F. (2020) The Current Status of Convalescent Plasma's Application in Acute Viral Infectious Diseases and Its Prospects in the Treatment of New Coronavirus Pneumonia. Chinese Journal of Biologicals, 1-5.

[21] Shanwell, A., Andersson, T.M.-L., Rostgaard, K., et al. (2009) Post-Transfusion Mortality among Recipients of ABO-Compatible but Non-Identical Plasma. Vox Sanguinis, 96, 316-323. https://doi.org/10.1111/j.1423-0410.2009.01167.x

[22] Inaba, K., Branco, B., Rhee, P., et al. (2010) Impact of ABO-Identical vs ABOCompatible Nonidentical Plasma Transfusion in Trauma Patients. The Archives of Surgery, 145, 899-906. https://doi.org/10.1001/archsurg.2010.175

[23] Berséus, O., Boman, K., Nessen, S.C., et al. (2013) Risks of Hemolysis Due to Anti-A and Anti-B Caused by the Transfusion of Blood or Blood Components Containing ABO-Incompatible Plasma. Transfusion, 53, 114S-123S. https://doi.org/10.1111/trf.12045

[24] Cao, W.C., Liu, W., Zhang, P.H., et al. (2007) Disappearance of Antibodies to SARS-Associated Coronavirus after Recovery. The New England Journal of Medicine, 357, 1162-1163. https://doi.org/10.1056/NEJMc070348 